\title{
The Political Culture of European Women in the Twenty-First Century
}

\author{
Dr. Dilina Beshiri \\ Department of Pedagogy, Aleksandër Moisiu University, Durrës \\ Email: dilina_b@hotmail.com \\ Prof. As. Dr. Edi Puka
}

Department of Education Sciences, European University of Tirana

Doi:10.5901/mjss.2014.v5n13p120

Email: edipuka@yahoo.it

\begin{abstract}
The relationship between women and politics continues to provoke discussions and is increasingly being addressed as a problem of democracy and not of women only. This research will deal with analyzing the relationship between political education and women, as well as the importance of educating to politics; the reason why there is need of developing in individuals and in women particularly the sense of individual and collective responsibility and the awareness of their rights in the common good, in order to teach them that the delegation people carry out through elections is of a representative and not an of a intellectual kind. In fact, despite significant successes in the growth of women's presence in education, employment and cultural life, we realize that the political participation of women is still very low. We will try to sensitize the public opinion on this issue, and change a political culture that still considers the man a legitimate protagonist of state management.
\end{abstract}

Keywords: women, policy, education, citizenship, culture.

\section{Introduzione}

La politica è un ambito in cui la presenza femminile è ancora molto scarsa; tuttavia, le donne che hanno frequentato i luoghi della politica, in qualità di capo di governo, hanno lasciato il segno tanto da essere soprannominate Ladies di ferro.

II rapporto tra donne e politica continua a far discutere e viene sempre più affrontato, come problema della democrazia e non solo delle donne. II taglio sociologico, consente - grazie anche all'ampiezza del materiale esaminato e proposto nel quadro di una problematica che resta aperta, senza vincoli ideologici e senza risposte conclusive - un approccio attento all'attualità della questione così come emerge nella vicenda politica odierna con i suoi dibattiti, i suoi condizionamenti, le sue delusioni. Al fondo di questa rivendicazione c'è più che un sacrosanto legittimo diritto soggettivo: c'è l'utopia della ricomposizione, nel progetto politico collettivo, delle sfere d'interessi dell'umanità, il riconoscimento del valore storico-politico chiave, per il disegno del futuro, dei problemi demografici e di quelli affettivi, del benessere quotidiano e dell'organizzazione della vita (Nicola, 1983: 11).

Nonostante, negli anni più recenti, la partecipazione politica delle donne abbia subito trasformazioni significative sia in merito alla quantità che alla qualità della partecipazione politica, diversi studi continuano a sottolineare che il network relazionale degli uomini continua ad essere non solo più esteso di quello delle donne, ma anche di più spiccata rilevanza politica. Tenuto conto del più basso livello di informazione politica e di un limitato senso di efficacia, per le donne l'inserimento in gruppi e strutture organizzative, anche se a carattere non specificamente politico, incrementa il senso di sicurezza nell'affermazione delle proprie opinioni ed incentiva la partecipazione politica, dal voto all'impegno attivo. La maggiore dipendenza dagli intermediari politici sarebbe la conseguenza di una bassa fiducia nella propria capacità di elaborare opinioni politiche autonome e della necessità di essere rassicurate. Questo bisogno di rassicurazione, che spesso fa capo a gruppi ed organizzazioni, ancora dominati da figure maschili, evidenzia il persistere del cosiddetto "gap di indipendenza".

La questione della scarsa presenza femminile nei luoghi di decisione politica è un dato che, a sessant'anni dall'acquisizione del diritto elettorale da parte delle donne, è sintomo di una sconfitta per la stessa democrazia e lascia aperto il problema del riequilibrio della rappresentanza e della realizzazione di una democrazia effettiva e non dimezzata.

\section{Emancipazione Femminile e Cultura Politica}

II Femminismo è stata la prima forma di identità pubblica che le donne, prima un' agguerrita minoranza, poi in gruppi 
sempre più estesi, si sono date a partire dalla fine del 1600. A quell'epoca si accese una accanita disputa in merito alle capacità e al ruolo sociale delle donne. II problema era all'ordine del giorno: le trasformazioni economiche, sociali e politiche avevano posto le premesse di una più ampia e consapevole partecipazione delle donne alla vita politica, artistica e culturale, eppure molte di loro conducevano ancora una vita grama e mortificante, escluse dai livelli alti dell'istruzione e da ogni ruolo significativo e consegnate, vita naturale durante, 0 al matrimonio 0 alla clausura.

Bisogna però aspettare fino alla Rivoluzione Francese (1789) per trovare documenti significativi sulla figura della donna. In Francia troviamo Olympe de Gouges (1748-1793), con "La dichiarazione dei diritti della donna e della cittadina", del 1791. L'intento della Dichiarazione era di rendere consapevoli le donne dei diritti che venivano loro negati e di chiederne quindi la reintegrazione affinché anche le donne divenissero delle cittadine a tutti gli effetti. La donna, secondo la Dichiarazione, nasce libera ed ha gli stessi diritti dell'uomo. Le distinzioni sociali possono essere fondate solo sull'utilità comune (art.1) (Santucci, 2004); i diritti naturali sono la libertà, la proprietà, la sicurezza e soprattutto la resistenza all'oppressione (art. 2); tutte le cittadine devono essere ammesse ad ogni dignità, posto e impiego pubblici secondo le loro capacità, e senza altre distinzioni che quelle delle loro virtù e dei loro talenti (art.6); la donna deve poter esprimere liberamente i suoi pensieri e le sue opinioni (art.11) (De Martino-Bruzzese, 1994: 181-183). In Inghilterra, proprio nello stesso periodo, c'è Mary Wollstonecraft (1739-1797), autrice di un'opera intitolata "Rivendicazione dei diritti della donna", (1792), che intende collocare le istanze di liberazione e di parità sociale e politica delle donne nel contesto del più generale programma illuministico dei diritti dell'uomo. Le donne devono uscire dalla loro gabbia dorata della "femminilità", che è l'altra faccia della emarginazione e della subordinazione; la donna deve acquisire l'ideale della ragione, rivolgendosi al suo stesso sesso; non più amanti seducenti ma mogli "affettuose e madri razionali". Le donne devono coinvolgersi pienamente nel progetto illuminista e riformatore : istruzione, diritti politici, responsabilità personale, parità economica, razionalità e virtù, libertà e felicità, sono questi gli ideali della Wollstonecraft, che giunge anche a proporre, provocatoriamente, una "castità" femminile demistificatrice dei rapporti ambigui con l'uomo. La mente femminile è stata relegata dalla tirannide maschile in un limbo di fatuità a cui le donne, per lo più, si sono adattate. Ma le donne oggi devono sapere che ai diritti che esse reclamano corrispondono dei doveri e che la ribellione contro il dominio maschile deve svolgersi in nome di valori universali, quali quelli emersi col pensiero moderno. Intelletto, virtù e libertà sono i tre volti della ragione illuminata che la Wollstonecraft. ha posto come principi del suo pensiero. II suo fine è la creazione di una "nuova civiltà" in cui l'umanità sia virtuosa e felice (Wollstonecraft, 1977).

In Italia il movimento per i diritti delle donne nacque in ritardo rispetto ad altri paesi; quando la rivoluzione industriale, cominciò ad occupare come forza lavoro anche le donne, si posero problemi quali l'orario di lavoro, da conciliare con il lavoro casalingo, e la tutela della maternità. Si formarono, così, dei gruppi femminili che all'inizio erano formati per lo più da donne della borghesia. In seguito si unirono a loro anche movimenti di donne socialiste. Pioniere del movimento per i diritti della donna furono, tra le altre, Anna Maria Mozzoni, con il suo libro autobiografico "Una donna ed Anna Kuliscioff'.

Il vero e proprio femminismo nascerà però solo nell'Ottocento. L'orizzonte etico e politico del femminismo ottocentesco è stato quello dell'egualitarismo fra i sessi e della emancipazione giuridica ed economica della donna. Nel corso dell'Ottocento le femministe si sono comunque impegnate, oltre che su obiettivi specifici, anche su tematiche riguardanti i diritti umani e civili in senso ampio: le lotte per la libertà di pensiero e di associazione, per l'abolizione della schiavitù e della prostituzione, per la pace. Altra cosa da notare è che il movimento femminista dall'inizio dell'Ottocento ha convissuto col movimento socialista.

Nella seconda metà dell'Ottocento il segno politico del femminismo cambiò in seguito ai processi di inurbazione e di industrializzazione che si svilupparono tanto in Europa quanto negli Stati Uniti. Non solo donne colte e altolocate ma anche donne del ceto operaio e piccolo-borghese furono coinvolte dal movimento socialista e da quello femminista che individuarono finalmente una strategia specifica per affrontare la "questione femminile".

Negli Stati Uniti ci fu Lucretia Coffin Mott (1793-1880), riformatrice sociale e predicatrice "quacchera", impegnata sui temi della abolizione della schiavitù, del voto alle donne e della pace nel mondo.

In Inghilterra si formò la "National Society for Woman's Suffrage", diretta da Lydia Becker , e la "Ladies National association" di Josephine Butler ,impegnata anche per una regolamentazione se non abolizione della prostituzione.

In Francia ricordiamo, Hubertine Auclert (1848-1914), che propose nel 1879 un salario per le casalinghe e si fece promotrice di uno sciopero fiscale delle donne fino alla concessione del diritto di voto. Nel 1904 fu protagonista di un episodio di cronaca che la rese ancora più celebre : nel corso di una ennesima manifestazione femminista, strappò una copia del Codice Napoleonico, di cui in quei giorni si celebrava l'anniversario, per le sue inique disposizioni, a detta della Auclert, in materia di diritto privato.

Dobbiamo però aspettare la metà degli anni '60 di questo secolo quando nacque il Movimento di Liberazione della Donna come espressione delle contraddizioni del ruolo sociale femminile nei paesi del capitalismo avanzato (Europa e 
USA); L'inserimento economico ai bassi livelli del sistema, sfruttamento del lavoro domestico, uso consumistico dell'immagine femminile, educazione repressiva, estraneità delle donne ai partiti politici tradizionali, esigenza di nuovi valori.

Durante questo periodo, precisamente nel 1963, apparve negli Stati Uniti un saggio di Betty Friedan, "La mistica della femminilità" (Betty Friedan, 1963), in cui sosteneva che nella società americana si era giunti al punto di massima tensione fra la realtà della vita femminile e l'immagine della donna proposta dai mass media e dalla cultura ufficiale. Secondo la Friedan, a partire dagli anni '40 era stata proposta una "mistica della femminilità" cioè un modello di vita e di felicità femminile organico e chiuso: amore, figli, marito, casa, acquisti, vestiti ecc. che finalmente entrava in crisi. ॥ dibattito si arricchì nel 1969 con la pubblicazione de, "La politica del sesso" di Kate Millet (Millet, 1979). Con questo libro si inaugurò la tendenza specificamente radicale del nuovo femminismo, una tendenza sessista e separatista che univa, alla fine analisi culturale e letteraria, il programma di una opposizione radicale contro la società, intesa anzitutto come società maschile e patriarcale. La Millet fece esplicitamente diventare l'azione del Femminismo una lotta contro un potere per un altro e diverso potere, per cui il fine del movimento non era più quello della parità, bensì quello della eccellenza della donna, della sua superiorità: era un femminismo della differenza, un femminismo che si qualificava soprattutto come movimento intellettuale.

Vent'anni dopo le prime manifestazioni di piazza e di lotte vincenti sul divorzio e l'aborto, il femminismo è ancora vivo, pur essendosi trasformato, nella cultura e nella riflessione politica delle donne (De Leo, Taricone, 1999: 265).

II femminismo radicale è diventato, di fatto, a partire dagli anni '80, un femminismo della complessità, un femminismo sistemico. Ma il fatto di essere diventato un femminismo colto, e per certi versi esoterico, non è stata la sola trasformazione. Da movimento di massa, da movimento sociale, è diventato una rete di piccoli gruppi: questo ha consentito un affinamento della elaborazione teorica ma forse ha fatto perdere posizioni sul piano politico, mentre i "diritti delle donne" sono diventati una sorta di luogo comune dell'agenda politica di tutti i partiti.

La sfida tentata dal movimento politico delle donne in Italia, di cui l'Unione Femminile Nazionale ha rappresentato l'espressione più articolata e matura, era in definitiva quella di assumere consapevolmente la parzialità di un soggetto escluso ad ogni livello dalla totalità di un progetto di cambiamento - le donne - come misura e paradigma universale della trasformazione.

Una simile prospettiva sarebbe stata sostenibile però soltanto se le donna fosse stata preparata a viversi come soggetto autonomo, che non assumesse il metro maschile per guardare al proprio ruolo nel mondo, ma che misurasse finalmente su se stessa (Buttafuoco, 1992: 27).

\section{La Partecipazione Politica e Sociale delle Donne}

Nonostante le donne, nel secolo scorso, abbiano acquisito i diritti politici, non sono riuscite ad entrare a far parte in misura consistente delle istituzioni politiche rappresentative. Questo fenomeno coinvolge tutti i Paesi del mondo indistintamente. I motivi di tale fenomeno sono diversi e legati sia alla crisi della rappresentanza, sia a dei fattori socioculturali. Si tratta di sensibilizzare su questo tema l'opinione pubblica, e modificare una cultura politica che, ancora oggi, considera l'uomo il legittimo protagonista della gestione dello Stato.

In più, occorrono delle misure concrete che promuovano la partecipazione politica delle donne. In primo luogo il sistema elettorale di tipo maggioritario, che di per sé tende ad escludere forze politiche minori, come ad esempio, anche i giovani, che oggi si trovano ai margini della politica. Nelle strutture politiche di base, come i partiti ed i sindacati, non essendo le donne presenti in misura considerevole, non si crea un movimento politico al femminile, capace di costituirsi come punto di riferimento per le elettrici e le elette.

Inoltre, l'assenza delle donne dalle organizzazioni partitiche primarie, priva la candidata alle elezioni, di un appoggio morale e politico durante la campagna elettorale.

II contatto con delle realtà, come la scuola, gli ospedali, la parrocchia, maturato durante la propria esperienza personale di figlia, moglie e madre, ha dato alle donne una singolare capacità di analizzare la società, per capirne i problemi e tentare di risolverli, spesso con successo. Mostrando un forte senso di responsabilità civica, le donne hanno scelto altre sedi politiche in cui operare.

A livello locale, in Italia, la presenza femminile è decisamente consistente, soprattutto se confrontata con i dati relativi al Parlamento; cresce moderatamente il numero delle donne presenti nelle Regioni e nelle Province, mentre nei Comuni si registra una significativa partecipazione femminile. È opportuno fare delle considerazioni sul perché le donne siano politicamente più presenti a livello locale, piuttosto che a livello centrale.

Nelle indagini recenti sulla partecipazione politica, almeno secondo gli indicatori prescelti, l'Italia risulta, essere il paese europeo nel quale la differenza tra uomini e donne è maggiore: lo scarto diminuisce decisamente tra i più giovani, 
ma è ancora consistente (Inglehart, 1988: 420-422).

L'accesso sempre più massiccio ai gradi alti della formazione scientifica e professionale è stato un fattore di messa in evidenza sempre più ampia della qualità della presenza femminile nella società e del rilievo dell'apporto che le donne possono fornire allo sviluppo civile del Italia. Ciò non è avvenuto senza fatica e senza incontrare ostacoli (Papa, 2010: 153). Ė sempre più chiaro che ovunque le donne abbiano intrapreso un percorso di formazione che doveva portarle a esiti professionali impegnativi e di responsabilità, ciò non poteva avvenire che pagando prezzi più alti, sensibilmente più alti rispetto agli uomini.

Tuttora comunque non c'è ancora la consapevolezza che la partecipazione della donna alla politica può portare quel pragmatismo e quella concretezza tipicamente femminile e la determinazione nel perseguire gli obbiettivi, con uno spirito di sacrificio e di dedizione al bene comune, in quanto meno preoccupate rispetto agli uomini della ricerca di posizioni di potere per una pura gratificazione personale, infatti le donne costituiscono la metà degli esseri liberi (Aristotele 1955: 60). La presenza delle donne in politica, in un ambito ancora prettamente maschile, potrebbe favorire perciò dei cambiamenti fondamentali sia a livello metodologico, sia nei contenuti del dibattito.

Una democrazia fondata sulla cittadinanza duale, di donne e di uomini, soggetti entrambi di diritto di cittadinanza e di rappresentanza. Non una quota da recuperare per le donne ma una garanzia di presenza al $50 \%$ tra candidate e candidati che, in modo alternato, devono comporre le liste elettorali, pena l'irricevibilità della lista stessa. Ed è questo l'altro aspetto sostanziale della proposta di legge, perché, finalmente, si rimette la politica tra uomini e donne sul piano pubblico, del confronto, e non la si costringe più nella strettoia del privato, della lotta tra i sessi. Perché non vi possono essere efficaci politiche a favore delle donne senza le donne protagoniste delle scelte. Perché non si promuove empowerment "in nome" delle donne ma "con" le donne (Donno, 2010: 148).

Avere più donne nelle assemblee elettive significa poter offrire uno stile di governo, a tutti i livelli, capace di interpretare i diversi bisogni e offrire risposte diversificate a tutti, uomini e donne, con una più aderente rappresentazione della realtà. Perché nessuna differenza sarà mai un valore se la realtà che ci circonda viene descritta e interpretata con un solo colore, un solo sguardo, da un solo sesso (Donno, 2010: 148).

\section{Le Donne in Europa: Gli Strumenti della Politica delle Donne}

Per analizzare il ruolo e la posizione delle donne europee nelle istituzioni politiche, è opportuno un breve cenno storico, partendo dall'acquisizione del diritto di voto, essendo, la storia politica femminile, recente.

Alla fine del XIII sec., le donne europee non godevano dei diritti civili né politici, concessi solo a frange ristrette della popolazione.

Infatti, prima di ottenere il diritto di voto e di eleggibilità sul piano cantonale e federale, le donne potevano esprimere il loro impegno politico solo nell'ambito di organizzazioni femminili e associazioni professionali. In alcuni paesi, potevano, inoltre, fare parte di commissioni scolastiche, commissioni per la pubblica assistenza, consigli ecclesiali e parrocchiali e qualche altra autorità politica. Anche le prime donne che ricoprirono cariche politiche comunali o regionali a cavallo tra gli anni Sessanta e gli anni Settanta si erano cimentate in tali consessi prima di avventurarsi sulla scena pubblica.

Nel 1971, quando le donne entrarono finalmente nella politica federale, incominciò anche la loro avanzata nelle funzioni di partito, nei parlamenti, negli esecutivi e in altre cariche politiche. Nel confronto europeo, la presenza femminile in Consiglio nazionale si situa oggi, con il 23.5\% conseguito alle elezioni federali del 1999, nella fascia mediana: percentuali più elevate si riscontrano nell'area scandinava e nei Paesi Bassi (tra il $36 \%$ e il 42.7\%), nonché in Germania (30.9\%), Spagna (28.3\%) e Austria (26.8\%). Nonostante i progressi compiuti e benché costituiscano la maggioranza della popolazione residente e dell'elettorato, le donne svizzere sono tuttora decisamente sottorappresentate a tutti i livelli politici.

I paesi nordici si situano tutti tra i primi dieci in graduatoria: negli ultimi cinque anni le prime 4 posizioni sono costantemente occupate da Islanda, Norvegia, Finlandia e Svezia (Global Gender Gap Index 2010), mentre la Danimarca si colloca al $7^{\circ}$ posto. I primi 4 paesi si trovano tra i primi 20 nel sottoindice della partecipazione e delle opportunità economiche e ottengono nuovamente i primi 4 posti nel sottoindice della partecipazione politica. Nei paesi nordici, infatti, la partecipazione femminile alla forza lavoro è tra le più alte al mondo e le differenze di salario nei confronti degli uomini sono tra le più basse al mondo, anche se non è ancora stata raggiunta la piena parità salariale. Le politiche di questi paesi includono congedi di paternità obbligatori in associazione a generosi congedi di maternità, incentivi e programmi di rientro post-maternità. In alcuni paesi il congedo può durare anche un intero anno: in Norvegia il congedo dura 46 settimane con una indennità pari al 100\% dello stipendio, o 56 settimane con l'80\% dello stipendio; in Svezia il congedo dura 480 giorni, con una indennità calcolata sulla base dello stipendio per i primi 390 giorni (''80\% dello stipendio, entro 
un tetto massimo) e una indennità fissa negli ultimi 90 giorni; in Danimarca dura 52 settimane all' $80 \%$ dello stipendio (con un tetto massimo). I programmi di rientro post-maternità islandesi hanno fortemente contribuito a posizionare il paese al primo posto nel Global Gender Gap Index: in Islanda vi sono il diritto legale di tornare al lavoro dopo la nascita del figlio e un sistema capillare di asili nido e scuole materne offerto da parte dei principali comuni. Le donne hanno inoltre molte opportunità di raggiungere posizioni di potere, anche perché i paesi nordici hanno adottato politiche di promozione della leadership femminile. Per quanto riguarda la partecipazione delle donne alla politica, i paesi nordici furono tra i pionieri del diritto al voto femminile e negli anni '70 i partiti politici norvegesi, svedesi e danesi hanno introdotto quote rosa su base volontaria, con un conseguente aumento della partecipazione politica femminile. Oggi i paesi nordici hanno percentuali di partecipazione politica femminile che si attestano tra le più alte al mondo, sia per quanto riguarda la percentuale di donne in parlamento (45\% in Svezia, 43\% in Islanda, 40\% in Norvegia e Finlandia, 38\% in Danimarca), sia per ciò che concerne il numero delle donne ministro (63\% in Finlandia, 53\% in Norvegia, $45 \%$ in Svezia e Islanda, 42\% in Danimarca).

Tab. 1 - Classifica mondiale della presenza femminile nei Parlamenti nazionali (2010)

\begin{tabular}{|c|c|c|c|c|c|c|c|c|c|}
\hline \multirow{2}{*}{ Rank } & \multirow{2}{*}{ Country } & \multicolumn{4}{|c|}{ Lower or single House } & \multicolumn{4}{|c|}{ Upper House or Senate } \\
\hline & & Elections & Seats & Women & $\% \mathrm{~W}$ & Elections & Seats & Women & $\% \mathrm{~W}$ \\
\hline 1 & Rwanda & 92008 & 80 & 45 & $56.30 \%$ & 102003 & 26 & 9 & $34.60 \%$ \\
\hline 2 & Sweden & 92010 & 349 & 157 & $45.00 \%$ & --- & --- & --- & --- \\
\hline 3 & South Africa & 42009 & 400 & 178 & $44.50 \%$ & 42009 & 54 & 16 & $29.60 \%$ \\
\hline 4 & Cuba & 12008 & 614 & 265 & $43.20 \%$ & --- & --- & --- & --- \\
\hline 5 & Iceland & 42009 & 63 & 27 & $42.90 \%$ & --- & --- & --- & --- \\
\hline 6 & Netherlands & 62010 & 150 & 61 & $40.70 \%$ & 52007 & 75 & 26 & $34.70 \%$ \\
\hline 7 & Finland & 32007 & 200 & 80 & $40.00 \%$ & --- & --- & --- & --- \\
\hline 8 & Norway & 92009 & 169 & 67 & $39.60 \%$ & --- & --- & --- & --- \\
\hline 9 & Belgium & 62010 & 150 & 59 & $39.30 \%$ & 62010 & 71 & 27 & $38.00 \%$ \\
\hline 10 & Mozambique & 102009 & 250 & 98 & $39.20 \%$ & --- & --- & --- & --- \\
\hline
\end{tabular}

Source: Inter-Parliamentary Union: http://www.ipu.org/wmn-e/classif.htm

Recentemente, il tema relativo alla partecipazione politica delle donne, è diventato molto attuale; l'UE ha intrapreso una serie di iniziative (sondaggi, indagini, dichiarazioni di principio, iniziative legislative), coinvolgendo anche i singoli governi nazionali,e le donne continuano ad essere sottorappresentate nelle posizioni che comportano responsabilità politiche ed economiche, anche se la percentuale è aumentata nel corso degli ultimi dieci anni. Il tasso di occupazione delle donne ance se è in crescita deve ancora aumentare per raggiungere l'obiettivo fissato dalla strategia Europea 2020.

\section{Come Aumentare la Partecipazione Femminile in Politica}

Varie sono le cause della bassa presenza di donne in politica. Innanzitutto la mancanza di tempo penalizza le donne per la difficoltà di conciliarne le richieste con l'attività di cura della famiglia e della gestione della casa. II problema diventa più grave quando a ciò si aggiunge la scarsità di mezzi economici che limita anche la possibilità di intraprendere campagne elettorali onerose e costose.

Per le donne il passaggio dal particolare al generale è essenzialmente passaggio dalla donna alle donne: dall'idea di se stessa come inerme per destino, alla scoperta di sé e delle altre insieme come oppresse (Gramaglia 1968: 179201).

La minore partecipazione politica delle donne ha cause multiple. Alle donne incombono, per esempio, quasi interamente le responsabilità famigliari e, pertanto, dei doveri sociali che le rendono meno disponibili per una carriera politica. Le donne accedono anche meno facilmente alla politica perché generalmente occupano posizioni meno alte degli uomini nella vita professionale e sono meno bene rappresentate nelle lobby economiche.

Quanto alle possibilità di profilarsi sulla scena politica, non hanno ancora modelli 0 una tradizione a cui ispirarsi. E infine molte donne preferiscono impegnarsi nelle istituzioni extraparlamentari o svolgere attività di volontariato in campo sociale 0 ecclesiale.

Ma è soprattutto la percezione della distanza tra il proprio mondo e quello della politica a tenere lontano le donne da questa attività: tanto le donne enfatizzano il valore ella concretezza, della necessità di misurare le proposte, di confrontarsi, costruire, lavorare per obbiettivi, tanto la politica appare loro uno spazio autoreferenziale, in cui si esercitano 
poteri e veti incrociati e di cui non sono chiari né gli obbiettivi, né le regole, né i luoghi di espressione (Zajczyk, 2007).

Anche il fattore temporale viene individuato da Gelli come un elemento che rende difficile alle donne l'accesso alla dimensione politica, nel senso che "manca una storia, mancano modelli di azione e di interventi/cambiamenti sulla realtà sociale ai quali le donne individualmente ed in gruppo possano fare riferimento. Manca la memoria di un potere che è esercizio della politica (Gelli, 2009). Nell'individuazione dei fattori che in altri paesi hanno ostacolato alle donne l'accesso alla politica ed alle istituzioni, Gelli, accanto alla "religione cattolica con influenza più o meno diretta sul modello della femminilità e del ruolo della donna in famiglia; al sistema maggioritario di voto"colloca al primo posto il "ritardo nella concessione del suffragio".

\section{Conclusioni}

Abbiamo visto che finora pregiudizi di tipo culturale hanno, infatti, relegato la donna all'ambito familiare oppure sono stati affidati loro ruoli mutuati dalla vita domestica e di scarsa rilevanza strategica. Per invertire questa tendenza bisognerebbe partire da una sorta di educazione e di sensibilizzazione sulle pari opportunità per l'uomo e la donna. Bisogna avviare iniziative volte a modificare una cultura, in cui l'uomo è considerato ancora il protagonista della gestione della repubblica ed attuare modalità di sensibilizzazione per favorire l'accesso alla politica di quella metà dell'umanità che, in quanto alla pari degli uomini, deve avere gli stessi diritti in nome di una rappresentanza più equilibrata e nel rispetto dei principi di democrazia e di uguaglianza.

Il compito più difficile è quello di promuovere attraverso azioni di sensibilizzazione una nuova cultura che riconosca i valori e l'indispensabilità del contributo delle donne nei processi decisionali. Deve essere un'azione volta non solo a favore delle donne, ma anche e soprattutto rivolta alle donne, perché prendano coscienza del ruolo che devono assumere nelle istituzioni.

La grande rivoluzione consiste innanzitutto nel modo di pensare delle donne, nel coraggio di presentarsi con la loro specificità, invece di fare propri i vecchi modelli maschili, mascolinizzando i propri comportamenti, nell'abbandonare lo scetticismo e nell'incominciare a prendere coscienza delle loro qualità e potenzialità con l'audacia, la convinzione e la capacità di cogliere le nuove esigenze e di agevolare il cambiamento.

\section{Bibliografia}

Aristotele, Politica e Costituzioni di Atene, Torino, Utet, 1955, trad. It, Viano C.A., (a cura di).

Betty Friedan, B., (1963). La mistica della femminilità, Milano: Edizioni di Comunità.

Buttafuoco, A., (1992). Per un diritto. Coeducazione e identità femminile nell'emancipazionismo italiano tra ' 800 e ' 900 , in Beseghi, E. \& Telmon, V. (a cura di), Educazione al femminile: dalla parità alla differenza, Firenze: La nuova Italia.

De Leo, M., Taricone, F., (1999). Quale politica per le donne, in Le donne in Italia. Diritti civili e politici, Napoli: Liguori Editore.

Donno, M. G., (2010). Cittadinanza duale e democrazia paritaria, in Etiche e politiche di genere, Bari: Servizio Editoriale Universitario.

Gelli, B., (2009). Psicologia della differenza di genere. Soggettività femminili tra vecchi pregiudizi e nuova cultura, Milano: Franco Angeli.

Gramaglia, M., (1968). II venir dopo e l'andar oltre del movimento femminista, in Quaderno Problemi del Socialismo, Roma: Fondazione Basso, pp. 179-201.

Inglehart, R., (1988). La nuova partecipazione bella società post-industriali, in Revista Italiana di Scienze politiche, XVIII, (3), Dicembre, pp. 420-422.

De Martino, Bruzzese, (1994). Le filosofe, Napoli: Liguori editore.

Millet, K., (1979). La politica del sesso, Milano: Bompiani.

Nicola, G. P., (1983). Donne e politica. Quale partecipazione? Roma: Città Nuova Editrici.

Papa, F., M., (2010). La questione della partecipazione politica delle donne. Una questione aperta della democrazia itiliana, in Etiche e politiche di genere, Bari: Servizio Editoriale Universitario.

Santucci, F., (2004). Donne protagoniste, Piombino: II Foglio.

Wollstonecraft, M., (1977). I diritti della donna, Roma: Editori Riuniti.

Zajczyk, F., (2007). La resistibile ascesa delle donne in Italia. Stereotipi di genere e costruzione di nuove identità, Milano: II Saggiatore. 